\title{
DISTANCIA, AFECTO Y RAZÓN: \\ ENTRENAMIENTO ELEMENTAL PARA ACTORES DE FEDERICO LEÓN Y MARTÍN REJTMAN
}

POR

ANTONIO GómeZ

Tulane University

La idea de teatralidad con frecuencia aparece en los estudios sobre el nuevo cine argentino o en declaraciones de algunos de sus directores o críticos como uno de los malos hábitos del cine nacional a lo largo de su historia, y muy particularmente en los años ochenta. En general se identifica con esa expresión un estilo afectado de actuación, con una clara propensión al histrionismo y al patetismo, y una desproporcionada dependencia en el texto pronunciado por los actores como único medio de articulación narrativa e ideológica. En gran medida puede describirse la aparición y progresiva diferenciación del nuevo cine argentino como una revolución en el ámbito de los estilos de actuación. Entre otras novedades, películas como Rapado (1992) de Martín Rejtman o Pizza, birra, faso (1998) de Caetano y Stagnaro exhibían con orgullo un método de dirección de actores que había superado exitosamente los vicios de la transferencia acrítica a la pantalla grande de técnicas de actuación del teatro, ya en forma directa, ya por la mediación de la producción televisiva -una retórica que por su parte pervivía en los noventa (y después) en el cine de Subiela, Aristarain o Mignona-. Sin embargo, es evidente que no había en los nuevos cineastas una mera resistencia al teatro y mucho menos una crítica. Se trataba de la necesidad de definir la especificidad de un método (o varios) de actuación que atendiera a las particularidades de la labor cinematográfica. Así, la fuerte prédica a favor de un estilo actoral menos dramático no implicó la disolución de las relaciones entre cine y teatro, sino más bien su fortalecimiento en la fijación de una nueva relación de complementariedad y colaboración que evitara anteriores situaciones de preponderancia de alguna de las artes.

El proceso puede iluminarse con un par de ejemplos. Un caso como Made in Argentina (1987) de Juan José Jusid es clara muestra de un proyecto teatral que da el salto hacia el cine solamente en función de alcanzar una audiencia más amplia e ingresar en otro ámbito de circulación, pero en que no puede advertirse una voluntad de intervención sobre el ámbito cinematográfico en sí, sino más bien el uso de su superestructura para promover una agenda política específica -lo que es posible que también sucediera con la exitosa puesta teatral de la obra-. En cambio, en una película 
como Los labios (2010) de Iván Fund y Santiago Loza, en que también se percibe la fuerte presencia de un componente teatral, la colaboración más fecunda e íntegra entre cine y teatro se pone al servicio de un mismo impulso experimental. En todo caso, en el cine actual de directores que son también directores de teatro (Santiago Loza, Iván Fund, Ana Katz, Federico León o Daniel Hendler, por ejemplo) el uso ancilar de lo teatral en el proyecto cinematográfico no implica la desvirtuación que se daba cuando la proporción bilateral era inversa. Lo mismo puede decirse para la puesta de obras teatrales en que la experimentación con elementos o soportes cinematográficos cumple una función importante (obras del mismo Federico León, Rodrigo Moreno o Lola Arias, por ejemplo).

En este contexto, Entrenamiento elemental para actores (2009) de Federico León y Martín Rejtman es al mismo tiempo un caso atípico y emblemático. Por un lado, nace en el marco de un proyecto específicamente concebido para dar lugar a la colaboración de un director de cine y uno de teatro, y da cuenta, por lo tanto, de una nutrida tradición de trabajo conjunto. Por otro lado, por su tematización explícita de esta problemática, por su manipulación de las especificidades genéricas y por su peculiar autorrefencialidad, es un producto que revisa desde una posición privilegiada la relación entre la tradición cinematográfica local y el teatro, y genera la situación de objetivación que nos proponemos analizar aquí.

En primer lugar, vamos a referirnos brevemente a la recurrencia de una retórica de la distancia en el nuevo cine argentino, que servirá de marco contextual de la posterior revisión y análisis del modo en que Entrenamiento elemental para actores produce y discute el efecto de distanciamiento -puntualmente, en la posible relación entre lo representado (la interacción entre un maestro de actuación y sus alumnos) y la propia retórica de la representación (que hace de la distancia entre la mirada y el objeto un punto de fuerza)-. Queremos luego terminar con una puesta en relación entre esta práctica y las proposiciones teóricas respecto del efecto de distanciamiento como recurso teatral.

No se puede limitar el nuevo cine argentino a una caracterización negativa -0 sea, al recuento de los rasgos que surgen como reacción a la tradición de la que quiere diferenciarse, y con los que propone la construcción de su propia entidad-, pero este es indudablemente un impulso importante en su configuración. Así puede explicarse el compromiso íntimo con el rasgo que queremos identificar como una "retórica de la distancia". Una de las piedras fundantes del nuevo cine argentino, Rapado, hace de la distancia un elemento sustancial de su poética: el primer efecto que la película logra imponer a la audiencia es el de la desconexión entre la mirada que articula el relato desde una exterioridad por momentos enervante, $y$ las alternativas vitales del personaje, al que nunca se pretende invadir en su interioridad. Esta ilusión de desconexión se

Revista Iberoamericana, Vol. LXXXII, Núm. 257, Octubre-Diciembre 2016, 793-804 ISSN 0034-9631 (Impreso)

ISSN 2154-4794 (Electrónico) 
hace luego extensiva a las relaciones entre los diversos personajes de la historia, entre los personajes y el espacio, entre los personajes y los objetos -eje que termina por dominar el relato y los siguientes proyectos de Rejtman-. La ilusión de distancia invade luego otros ámbitos y se insinúa como la relación impuesta entre los personajes y los actores que les dan cuerpo -rasgo que se advierte principalmente en su dicción "desdramatizada"-, para ulteriormente proponerse como modelo de la relación que el espectador debe establecer con la historia que se desarrolla en pantalla.

A partir de Rapado podría hablarse de una fructífera tradición de distancia en el cine nacional, que si bien podría rastrearse en casi toda la producción comúnmente asociada a la categoría de nuevo cine argentino, tiene algunos ejemplos especialmente notables. Siguiendo la propuesta poética de Rejtman, que vincula tradiciones tan inconexas como las estrategias narrativas de la comedia y la retórica de cámara del cinéma verité, la propuesta ha sido tensada casi hasta puntos extremos por cineastas como Ezequiel Acuña o Celina Murga. En esta línea, la imposición de "distancia"-sobre todo a nivel de la dirección de actores- ha tenido un efecto de purga que finalmente ha revalorizado y cargado de nuevo sentido el uso discreto de ciertos picos dramáticos. Es el caso, por ejemplo, de las películas de Lucrecia Martel. Por otro lado, inscribiéndose en un registro más experimental para borronear los límites entre ficción y documental, el cine de Lisandro Alonso está concebido como una profunda reflexión en torno al ejercicio de la distancia. Sus cuatro primeros largometrajes ponen en práctica la misma estrategia para la construcción de la imagen: el registro exterior, casi en tiempo real, de un período de la vida de un personaje (encarnado por un "actor natural", o sea, un actor no profesional que se identifica de cerca con el personaje, por ejemplo, por ciertas capacidades específicas: cortar un árbol, carnear una cabra, etc.) en relación con su ambiente. Como se ha dicho acerca de uno de estos personajes, la separación entre la mirada y el objeto es tal que "nada sabemos de lo que piensa, siente o imagina" (Martins) -los personajes están limitados a su pura exterioridad y definidos por la distancia entre ellos y la cámara que los registra, el cineasta que los construye y el espectador que los consume-. El impacto mayor de la línea profundizada por Alonso, que es fácil rastrear tanto en el ámbito del documental (Criada, de Matías Herrera Córdoba) como en el de la ficción (Las acacias, de Pablo Giorgelli), ha terminado por volver al cine de Rejtman. Su documental Copacabana de 2006 -también, como Entrenamiento elemental para actores, un proyecto para la televisión- es una especie de ensayo sobre la distancia en el documental de observación. No solo en el ámbito técnico y retórico, sino también en su revisión de los presupuestos del cine etnográfico (Gómez). En Copacabana, como en el cine de Alonso, aun si responden a estímulos muy diversos, la retórica de la distancia se funda en el redescubrimiento del espíritu de la curiosidad, por un lado, y la ilusión de que la mínima intervención de la mirada puede preservar el carácter genuino del objeto, por otro. Pero esta combinación, cuya base es netamente racional, termina por convertirse en una postura ética (la voluntad

Revista Iberoamericana, Vol. LXXXII, Núm. 257, Octubre-Diciembre 2016, 793-804 ISSN 0034-9631 (Impreso)

ISSN 2154-4794 (Electrónico) 
de respetar la integridad del objeto que existe antes de su conversión en representación) $\mathrm{y}$, eventualmente, en una actitud afectiva: no cuesta advertir detrás del gesto obsesivo con que la cámara sigue a Misael en La libertad de Lisandro Alonso, por ejemplo, un acto de cariño - una vinculación afectiva que rápidamente se traslada al espectador-.

\section{LA PEDAGOGÍA DE LA DISTANCIA}

Las secuencias iniciales de Entrenamiento elemental para actores le tienden al espectador una trampa inocente que sirve de clave para acceder al resto del filme. Se suceden tomas de niños en acciones cotidianas, algunas quizás un poco inusuales, pero igualmente previsibles en la rutina de un niño: cepillarse los dientes frente al espejo, escabullirse fuera de la casa por la noche y espiar hacia el interior, acomodar y ocultar cosas en una habitación, observar a los padres dormidos, arrastrar una silla y sentarse junto a una ventana, ducharse, jugar a "cuerpo a tierra", rezar. Los primeros minutos de la película no plantean otra continuidad narrativa que la identificación etaria entre los personajes, y se propone así una acumulación de diferentes anécdotas de niños, pero sin conexión entre ellas. A los pocos minutos, sin embargo, el espectador entiende que lo que une a estos niños es su condición de actores y de compañeros de la clase de actuación que se convierte en el tema de la película. El estudio en que se desarrolla la clase es asimismo el escenario dominante del resto del filme, con lo que estas breves escenas fuera de ese espacio resultan, en visión retrospectiva, intrigantes: ¿se trataba de escenas actuadas? ¿Eran ejercicios para hacer fuera de la clase? ¿O eran efectivamente momentos legítimos de la vida real de los actores?

Ahora bien, la trampa se encuentra en el tramo final de esa primera colección de escenas: la niña con un brazo enyesado que le reza a la imagen de Cristo y deja caer lágrimas mientras murmura en voz muy baja, cada vez más emocionada, no es una de las alumnas de la clase de actuación, sino el personaje de otra película. Si bien la secuencia de esta película se inserta en Entrenamiento elemental para actores sin marcas de su condición de cita, a los pocos segundos hay un corte a un plano más amplio en que vemos que la escena transcurre en la pantalla de un televisor, a cuyo lado hay otra niña imitando la situación. Se escucha entonces una voz que comenta la técnica de la actriz en pantalla para producir el llanto: "Miren cómo relaja los ojos. La tensión que pone en la boca. Pone los ojos fuera de foco y eso permite que el lagrimal se dilate. ¿Lo ven?". Quien habla es Sergio, el maestro de teatro, y se comprende entonces que este es un ejercicio de su clase, que el profesor da por finalizado cuando la alumna logra a su vez llorar.

Este comienzo engañoso sugiere la duplicidad que se impone a lo largo de toda la película: nos encontramos en un universo de simulación en que se van a exhibir y analizar no solamente los modos de producir la verosimilitud, sino también las estrategias para provocar reacciones emotivas en el espectador. El desenmascaramiento de la

Revista Iberoamericana, Vol. LXXXII, Núm. 257, Octubre-Diciembre 2016, 793-804 ISSN 0034-9631 (Impreso)

ISSN 2154-4794 (Electrónico) 
técnica para llorar en la escena citada -que proviene, además, de un reciente ejemplo consagrado de película con niños: Ponette de Jacques Doillon-nos pone sobre aviso del carácter artificial de la representación, reduciendo a manipulaciones del cuerpo lo que a primera vista se nos presenta como la irrupción de lo incontrolable del espíritu. Pero también nos alerta sobre la vulnerabilidad del espectador, en quien este gesto artificial genera (o tiene la potencia de generar) una reacción emotiva real. De hecho, hay un corte a uno de los compañeros que está viendo el ejercicio, que también ha llorado. Inmediatamente después del éxito de la alumna en producir las lágrimas, la película exhibe con cierto énfasis un elemento más grosero, más mecánico, de la imitación de la escena de pantalla: la niña se quita el yeso falso de su brazo y se lo cede a la que debe hacer el ejercicio a continuación. El yeso, el llanto y el dolor que lo produce son ficcionales, tanto en Ponette como en Entrenamiento elemental para actores. Tan artificiales como las lágrimas de Ponette son las de la alumna de Sergio -la hemos visto en plena tarea, siguiendo las indicaciones de su maestro-, pero tenemos que comprender, finalmente, que también lo son las del compañero que observa el ejercicio y que se nos proponen a nosotros, observadores del niño que observa el ejercicio, como reales. La clase de teatro es ficcional, y los niños que representan a los estudiantes de actuación son, por lo tanto, actores. Resulta imposible no atender a esa recursividad, porque el discurso central de la película tiene que ver con la ética de la actuación infantil.

Los principios de la dirección de actores de la película no tienen por qué corresponderse con los que imparte con un rigor casi violento Sergio-y la posibilidad de un choque entre estas dos instancias pone a la película en una situación privilegiada para discutir los conflictos entre una estética de la verosimilitud y una ética de la emoción-. La noción de distancia -o las diferentes nociones asociadas a esa palabra y algunos de sus derivados en la teoría teatral y cinematográfica- resulta el mejor referente para atender a la propuesta de la película. Como es ya casi un lugar común al hablar del cine de Rejtman, se puede advertir en esta película una distancia muy calculada entre la mirada de la cámara y los objetos observados. Las relaciones entre los personajes, su vinculación con el espacio y la articulación misma de la acción están narradas con la mínima participación de la mirada del narrador -y la película adopta por momentos una retórica visual muy semejante a la de un documental de observación (con lo que a veces ha sido confundida)-. La concreción más clara de la distancia tiene que ver, sin embargo, con la poca naturalidad del discurso de Sergio, en la interpretación de Fabián Arenillas. Los parlamentos de Sergio imponen distancia desde distintas perspectivas: el ritmo con que son pronunciados es demasiado acelerado, la estructura sintáctica es propia de la lengua escrita, su léxico es muy complejo, no hay interés en establecer una comunicación efectiva, imponen un tono de autoridad desmedida. El choque más significativo se evidencia cuando se tiene en cuenta la audiencia natural de esos parlamentos: los niños de la clase. El personaje ha sido construido especialmente en

Revista Iberoamericana, Vol. LXXXII, Núm. 257, Octubre-Diciembre 2016, 793-804 ISSN 0034-9631 (Impreso)

ISSN 2154-4794 (Electrónico) 
su uso de la lengua, aunque al mismo tiempo ese uso absolutamente enajenado de la lengua, sin relación alguna con un referente real, es lo que a su vez revela a Sergio exclusivamente como la construcción de una técnica de actuación, y lo separa del actor que lo encarna. Su primer parlamento es ejemplar: unas dos páginas de crítica sobre los modos tradicionales de dirigir a niños actores, pronunciadas en ochenta segundos. Por su profunda falta de realismo, Sergio se convierte más en una función que en un personaje, y su método de enseñanza en un comentario sobre la naturaleza de la representación.

La película cuenta con numerosas reflexiones de Sergio sobre el modo en que los niños deben desarrollar su capacidad de actuar, todas planteadas con el mismo nivel de objetividad y exterioridad: son presentadas verbalmente por Sergio como verdades que no admiten discusión. Su autoridad es absoluta. Así, en una especie de ejercicio de introspección, con un fondo de música serena, dice:

En este ejercicio generalmente las mujeres lloran. Cuando uno llora tiene que aprender a capitalizar ese estado, tomar distancia para poder manejarlo. Poder ver el recorrido que hice para llegar a ese estado para después utilizarlo en otro momento. Todo sirve. Tenemos que entrenar una mirada externa sobre nosotros mismos.

Sin duda esta idea contrasta con el primer ejercicio al que nos referimos, en que el llanto no es alcanzado como un estado interior, sino producido por medios mecánicos artificiales. Estamos frente a una nueva deconstrucción del efecto de verosimilitud en la película: aunque Sergio es presentado como el creador y defensor de un método riguroso de actuación, sus prácticas están llenas de contradicciones, porque en rigor ese método no existe más que en el universo ficcional del filme. De todos modos, lo más interesante es que en este ejercicio en particular está tratando de convocar metodologías tan contrastantes que termina por caer en una suerte de absurdo: acá se plantea la introspección como una vía para el distanciamiento del sujeto sobre sí mismo. La frase despierta ciertos ecos de la propuesta brechteana de distanciamiento, efecto de distanciamiento o efecto de extrañamiento - como ha sido referido en español el original alemán Verfremdungseffekt - que literalmente podría parafrasearse como "el efecto de convertir en extraño o ajeno". En resumen la propuesta de Brecht para renovar el teatro como hecho estético y de consumo consistía en el rechazo categórico de la noción aristotélica de "empatía", sistematizada en su Poética. Si en la interpretación de Aristóteles el actor y el texto sirven de vehículo para la identificación entre el público y el personaje en escena, de modo que el espectador haga suyo el drama emocional

Véanse los siguientes textos de Brecht: "The epic ..." en Willett 22-4; "Alienation ..." (91-9); "Short ..." 136-47; Jameson, Brecht and Method 35-42; Ciria, "Brecht y nosotros".

Revista Iberoamericana, Vol. LXXXII, Núm. 257, Octubre-Diciembre 2016, 793-804 ISSN 0034-9631 (Impreso) 
del personaje y pueda así ser purificado por la experiencia vicaria de su sufrimiento inmerecido en el proceso de catarsis, la propuesta brechteana quiere romper ese lazo de identificación y remplazarlo con una actitud de objetivación: el público debe asimilar el espectáculo teatral como efectivamente diferente de la propia experiencia para afrontar lo representado con un espíritu analítico, y no con una actitud de compasión. El foco se mueve de una perspectiva emocional, afectiva, a una perspectiva racional; y de una función individual, religiosa, a otra social, política. La reflexión de Brecht se concentra entonces en la necesidad de promover una nueva escuela de teatro en que el actor aprenda las técnicas necesarias para producir en su público el efecto de distanciamiento. En palabras de Brecht: "El sostén esencial del teatro épico es quizás que apela menos a los sentimientos que a la razón del espectador. En vez de compartir una experiencia, el espectador debe internalizar su comprensión de las cosas" (Willett 23, traducción mía).

Pero evidentemente este no es el concepto de distancia que maneja Sergio en el parlamento anterior, en que se refiere a una especie de enajenación del actor respecto de su propio mundo afectivo, para poder observarlo desde afuera y reproducirlo a conciencia. Es también una propuesta racional, pero distinta de la cifrada en el ejercicio de imitación de Ponette. Aunque, como ya hemos dicho, sistematizar el método de Sergio sería desatender a la propuesta misma de la película, hay algunos elementos insistentes que pueden recuperarse para caracterizar la propuesta ética y estética de estas clases de actuación, y compararlas así con las que se puede suponer que sustentan la dirección de actores en la película. ${ }^{2}$ En primer lugar, Sergio abomina del histrionismo. La película sigue en un momento las alternativas de una alumna nueva, Sofía, que es caracterizada como "histriónica" y a la que en un momento Sergio excluye de la clase para recomendarle prácticas que sirvan mejor a su expresividad desbordada. El aprendizaje de la actuación no parece ser, para Sergio, la resolución de una inclinación vocacional. En segundo lugar, no entiende la actividad del taller como pública, y se niega por lo tanto a organizar muestras o exhibiciones del progreso de los estudiantes. Finalmente, apoya todas sus decisiones sobre el trabajo con los niños (entre ellas, las dos anteriores) en un ideario ético estricto que busca oponerse a los malos modos tradicionales de vincular a los niños con la actuación. En general, su propio método recupera una base de respeto por el niño, a quien nombra, por ejemplo, no como "niño actor" sino como simplemente "actor". La película plantea, sin embargo, una situación que bordea el absurdo, y cuya carga humorística estriba en un contraste. "El humor surge del contraste entre alguien que tiene un método de enseñanza muy serio, y que quienes lo ejecutan son chicos”, explica Rejtman (Segal 28).

2 En Lerer se advierte que la codirección entre Rejtman y León no cayó en la división de roles de puesta en escena y dirección de actores, respectivamente, sino que hicieron un trabajo integral en conjunto.

Revista Iberoamericana, Vol. LXXXII, Núm. 257, Octubre-Diciembre 2016, 793-804 ISSN 0034-9631 (Impreso)

ISSN 2154-4794 (Electrónico) 
Las fuerzas que regulan el trabajo de Sergio tienen que ver, también, con la distancia, con la separación: separar su escuela de teatro de los usos habituales, convencionales, reducidos a consumo; usar la actuación como un modo de distanciarse de los demás, casi como una práctica secreta; convertirla en un acto extraño, distinto de lo que se conocía hasta ahora. Mediante mecanismos diversos la acción de Sergio parece apuntar a los mismos resultados que los propuestos por Brecht: una actitud reflexiva sobre el acto de representación. En el universo en que se maneja Sergio, eso es lo que la actuación provoca en los niños de su clase (y en un momento, también en sus padres, que vienen a quejarse de la ausencia de pruebas del aprendizaje de sus hijos y terminan también ellos como alumnos de la clase) -pero no, claro, en el público inexistente-.

Hay dos grandes diferencias entre el método de Sergio y las premisas que podemos reponer en la dirección de actores de la película. En primer lugar, que tanto el raro y tenue efecto humorístico que se percibe de principio a fin como la articulación del personaje central, el profesor, dependen de algún modo de la capacidad histriónica de Arenillas, que se impone más allá de la neutralización exigida por algunos gestos de la dirección: el ritmo, la velocidad y el tono inexpresivo de su dicción -elemento que no puede haber sido descuidado por Rejtman, siempre atento a cómo suenan sus diálogos. En segundo lugar, que el rigor obsesivo de Sergio está llevado en la ficción a un punto tal que no podría encontrar parangón en ningún director que efectivamente tratara con niños - como los directores aclaran en varias entrevistas-. Por lo demás, se puede suponer que Rejtman y León comparten con su personaje ciertas estrategias y sobre todo la postura ética respecto al niño como artista y creador. Ahora, cabe preguntarse por su alineamiento en la oposición entre una apuesta por lo emocional o por lo racional como expectativa de trabajo, o, lo que es lo mismo, por el valor otorgado a la distancia que se imprime en todo el filme. Si no se trata de la distancia como aislamiento y diferenciación, como parece proponerla el personaje al mundo interno de la ficción, ni del extrañamiento provocado por la ruptura voluntaria del efecto de ficción para disparar el mecanismo reflexivo en el espectador, ¿qué función cumple la distancia que se presenta en Entrenamiento elemental para actores, por un lado, en la objetivación de lo representado no mediante técnicas actorales, sino por mecanismos de la puesta en escena, y por otro, en la premisa básica de la representación fotográfica de la realidad, cuya tecnología exige una distancia mínima entre objeto y cámara, su desidentificación? Hay sin duda la transferencia del ideario brechteano de enajenar un objeto, exhibirlo a una nueva luz y analizarlo desde una posición puramente racional. Es lo que la película hace en varios niveles: en los más obvios, con la pedagogía como práctica social y con la infancia como estado preconcebido, regulado y previsible. Pero también, en un plano más interesante, con los hábitos de consumo del espectador, con la construcción de lo verosímil, y con la generación de efectos emocionales -el modo ulterior de producir "realidades"-.

Revista Iberoamericana, Vol. LXXXII, Núm. 257, Octubre-Diciembre 2016, 793-804 ISSN 0034-9631 (Impreso) 
Así, la película se inscribe en el proceso de distanciamiento tal cual lo describe Fredric Jameson (vía Roland Barthes) en su ensayo sobre Brecht. Al deslindar las diferentes interpretaciones del efecto de distanciamiento -que rebautiza "V-effect"Jameson advierte cuatro tendencias: primero, la más literal, hacer que algo se vea extraño; luego la meramente técnica, los modos de lograr que algo se vea extraño -y destaca aquí que Brecht no limitaba sus observaciones solo al teatro-; en tercer lugar, el proceso de resistencia a la identificación aristotélica; y finalmente:

...la cuarta y final formulación del $V$-effect, ulteriormente política, que subsume a todas las descripciones anteriores y las expone también a una nueva luz. En ella lo familiar o habitual es reidentificado como "natural", y su extrañamiento levanta el velo de esa apariencia, que sugiere a su vez lo permanente y lo eterno, y muestra que en cambio el objeto es "histórico", a lo que puede agregarse como corolario político: hecho o contruido por seres humanos, y por lo tanto suceptible de ser cambiado por ellos, o incluso remplazado. (40, traducción mía)

Si una aproximación racional hacia la representación -lograda mediante la producción del efecto de extrañamiento- tiene en última instancia un poder subversivo, ¿debemos suponer que desde la perspectiva aristotélica, frente a un arte que trabaja por la aproximación afectiva a que conduce el ciclo empatía-identificación-catarsis, no se puede llegar a esta movilización? Resulta imperativo reconocer que el modelo aristotélico también es político, pero en el sentido inverso. La tragedia funcionaba en el contexto de la Atenas clásica -al igual que todas las otras artes, excluyendo quizás a la comedia- como una estructura de reproducción ideológica y, por lo tanto, como una herramienta para perpetuar el statu quo. El proceso mismo de la catarsis, que suele ser limitado a su relación con el discurso religioso, es también un medio de contención social: en su dimensión pedagógica, la catarsis suponía la incorporación del miedo y se convertía así en un eficaz recurso para impedir de cualquier impulso por alterar el orden constituido. Una reacción como la de Brecht cobra entonces un sentido más claro:

Necesitamos un tipo de teatro que no solo despliegue los sentimientos, percepciones e impulsos posibles dentro del campo histórico particular de las relaciones humanas en el cual tiene lugar la acción, sino que emplee y fomente dichos pensamientos y sentimientos para que ayuden a transformar el propio campo. (Ciria 13)

Ahora bien, ¿es posible pensar en una retórica de la distancia que no excluya lo afectivo, que sea también la expresión de un contenido emocional? ¿Es acertado asimilar distancia con ausencia? Otra secuencia de la película - u otro de los ejercicios de la clase de Sergio- ayuda a discutir este punto. Uno de los alumnos le pregunta: "¿Ya estamos actuando, profesor, en todo lo que hacemos?" Sergio le devuelve la pregunta: “Estás actuando ahora?” y propone como ejercicio la reiteración idéntica de toda

Revista Iberoamericana, Vol. LXXXII, Núm. 257, Octubre-Diciembre 2016, 793-804 ISSN 0034-9631 (Impreso)

ISSN 2154-4794 (Electrónico) 
la escena, ahora actuando: el alumno que preguntó hace de sí mismo, y otro alumno hace de profesor. Repiten el intercambio, y con ello se vuelve a crear la situación que se creaba con Ponette: la conversión de la escena real en una escena actuada le da un nuevo sentido (o sinsentido) a las preguntas “ ¿ya estamos actuando?” “ “¿estás actuando ahora?”, pero también señala el carácter ficcional de la escena original y por lo tanto vuelve a imponer el efecto de extrañamiento sobre el espectador. La conclusión de Sergio es algo trivial: "A veces no sabemos cuando estamos actuando", pero antes de esto justifica así el ejercicio de repetición: "Hay que retirarse. A veces el actor cree que si no está presente no está actuando". Esta defensa del "retirarse" como modo de lograr una actuación más efectiva puede aplicarse al plan mismo de la película: aun en la separación se puede estar actuando, aun en el distanciarse se puede estar estableciendo un compromiso afectivo con el objeto.

Hemos sugerido aquí al menos dos modos en que la imposición de distancia entre la mirada y el objeto puede suponer la fusión no contradictoria entre razón y emoción, que quisiéramos ahora sistematizar a modo de conclusión. En primer lugar, hay que reconocer en este acto la voluntad de no trivializar lo emotivo, de dejarlo fluir de la imagen y de la relación que el espectador puede establecer con la imagen. Siguiendo la sugerencia de Sergio, de ese mismo retirarse puede surgir la relación afectiva más profunda. Y esto nos devuelve al ejercicio de Ponette, porque en definitiva la observación de una imagen desde la distancia se asemeja mucho al acto de veneración, que es el que se lleva a cabo en la escena de la película francesa. La niña del filme -no viene a cuento ahora en qué circunstancias- está arrodillada frente a una imagen de Cristo. En el ejercicio de la clase hay en cambio una imagen de la virgen, pero la alumna no está solamente arrodillada frente a ella, sino también frente al televisor con la imagen de Ponette -a quien rinde, de algún modo, veneración-. O sea, establece con esta imagen de la que se encuentra separada, distante, con este elemento que está siendo objetivado al punto de enajenarlo de su contenido narrativo (como ahora, poco importa en la clase por qué llora Ponette), una relación fuertemente emotiva. El acto de venerar propone así un modo de relación con la imagen que aúna cercanía afectiva con distancia objetiva. La película convierte en extraño, una vez más, un acto normal: el que lleva a cabo en su momento el espectador.

En segundo lugar, también el respeto (una ligera variante de la veneración) es una expresión afectiva. Si se la despoja de sus derivaciones humorísticas -el contenido emocional más a flor de piel en esta película-, Entrenamiento elemental para actores utiliza la distancia como un modo de expresar su respeto por el acto que representa. $\mathrm{Y}$ ese respeto implica no solo una valoración positiva de lo que se encuentra frente al objetivo de la cámara, sino también una articulación intelectual, un modo de conocimiento. La combinación de curiosidad y respeto es, de nuevo, la clave con qué explicar eficientemente lo que se está llevando a cabo en la construcción de la clase

Revista Iberoamericana, Vol. LXXXII, Núm. 257, Octubre-Diciembre 2016, 793-804 ISSN 0034-9631 (Impreso) 
de actuación -así como lo era en el caso de La libertad: el análisis de un oficio y de su ejercicio-- Misael en la película de Alonso, y Sergio en ésta, son representados en tanto trabajadores. Su condición de trabajadores es, mediante la distancia impuesta por la representación cinematográfica, enajenada, convertida en un objeto que se ofrece al análisis racional, pero no por eso despojada de contenido afectivo. Por el contrario, la actitud que parece primar en estos acercamientos es precisamente el interés, el apego. Quizás por eso resulte pertinente la última secuencia, en que Sergio se ofrece a sus estudiantes como un objeto diferente del que conocen hasta entonces. La secuencia final del filme es un video del profesor en situaciones cotidianas, en su casa, en la calle, con su novia, bailando en una fiesta, cocinando. Es un reflejo de la secuencia inicial del filme -hasta aparece también cepillándose los dientes- sólo que en este caso no hay ambigüedad acerca de su formato: es un video casero que están viendo en clase. Sergio lo introduce así: "Es bueno que ustedes me puedan ver fuera de las clases, en mi vida cotidiana. No hace falta que les diga que esta es una selección". Una nueva puesta en abismo que termina por retomar de un modo absurdo las revelaciones hechas en la clase -y en la película-: no hay afuera de la actuación, todo lo representado es ajeno, no hay alternativas a la apreciación afectiva de la realidad.

\section{BiBLIOGRAFÍA}

Ciria, Alberto. "Brecht y nosotros". Brecht. Alberto Ciria y Daniel Divinsky, eds. Buenos Aires: Jorge Álvarez, 1967. 7-19.

Entrenamiento elemental para actores. Federico León y Martín Rejtman, dirs. Argentina, 2009.

Gómez, Antonio. "Argentine Multiculturalism and the Ethnographic Shift in Documentary Cinema: Martín Rejtman's Copacabana." Social Identities 19/3-4 (August, 2013): 340-355.

Jameson, Fredric. Brecht and Method. London: Verso, 1998.

León, Federico y Martín Rejtman. Entrenamiento elemental para actores. Buenos Aires: La Bestia Equilátera, 2012.

Lerer, Diego. "De distintas fuentes". <http://edant.clarin.com/diario/2009/03/29/ espectaculos/c-01001.htm>. 3 ago. 2016.

Martins, Laura. "Cine, política y (post)estado. La libertad de Lisandro Alonso". $<$ http:// nuevomundo.revues.org/58374?lang=en>. 3 ago. 2016.

Pellettieri, Osvaldo, ed. De Bertolt Brecht a Ricardo Monti. Teatro en lengua alemana y teatro argentino 1900-1994. Buenos Aires: Galerna, 1994.

Ponette. Jacques Doillon, dir. BAC Films, 1996.

Rapado. Martín Rejtman, dir. Epicentre Films, 1992.

Segal, Guido. "Reconfiguraciones. Entrevista con Martín Rejtman y Federico León”. El Amante 208 (sept. 2009): 27-8.

Revista Iberoamericana, Vol. LXXXII, Núm. 257, Octubre-Diciembre 2016, 793-804 ISSN 0034-9631 (Impreso)

ISSN 2154-4794 (Electrónico) 
Willett, John, ed. Brecht on Theatre. The Development of an Aesthetic. [1964]. New York: Hill \& Wang, 1992. 